\title{
CONNECTEDNESS IN SOME TOPOLOGICAL VECTOR-LATTICE GROUPS OF SEQUENCES
}

\author{
LECH DREWNOWSKI and MAREK NAWROCKI*
}

\begin{abstract}
Let $\eta$ be a strictly positive submeasure on $\mathrm{N}$. It is shown that the space $\omega(\eta)$ of all real sequences, considered with the topology $\tau_{\eta}$ of convergence in submeasure $\eta$, is (pathwise) connected iff $\eta$ is core-nonatomic. Moreover, for an arbitrary submeasure $\eta$, the connected component of the origin in $\omega(\eta)$ is characterized and shown to be an ideal. Some results of similar nature are also established for general topological vector-lattice groups as well as for the topological vector groups of Banach space valued sequences with the topology $\tau_{\eta}$.
\end{abstract}

\section{Introduction}

By a topological vector-lattice group (TVLG) we mean a real vector lattice $E$ equipped with a Hausdorff topology $\tau$ such that $(E,+; \tau)$ is a topological lattice group (with uniformly continuous lattice operations). In other words, $(E,+; \tau)$ is required to be a topological group with a base of zeroneighborhoods consisting of solid (hence also balanced) subsets of $E$. In particular, $E$ is then a (locally balanced) topological vector group (TVG). For more information (and references) on TVG's, see [2] and [4]; for topological vector lattices (TVL) (or locally solid Riesz spaces), see [1].

The topology of a metrizable TVLG $E$ can always be defined (in the usual manner) by a monotone $F G$-norm, that is, a functional $\|\cdot\|: E \rightarrow[0, \infty]$ such that: $\|x\|=0$ iff $x=0 ;\|x+y\| \leqslant\|x\|+\|y\| ;\|s x\| \leqslant\|t x\|$ whenever $|s| \leqslant|t|$; and $\|x\| \leqslant\|y\|$ whenever $|x| \leqslant|y|$. For a TVG $E$, we denote

$$
v(E)=\left\{x \in E:(\tau)-\lim _{t \rightarrow 0} t x=0\right\},
$$

$c(E)=$ the connected component of the origin in $E$.

Then $v(E)$ is the largest topological vector subspace of $E, v(E) \subset c(E)$, and both $v(E)$ and $c(E)$ are closed vector subspaces of $E$ (cf. [2, Rem. 2.4], and

\footnotetext{
* This research was partially supported by The Ministry of Science and Higher Education, Poland, Grant no. N N 201274033.

Received 17 December 2008, in revised form 31 March 312009.
} 
the proof of Theorem 2.2 below). If $E$ is a TVLG then, obviously, $v(E)$ is a TVL and an ideal in $E$ and, as we show in this paper, also $c(E)$ is an ideal in $E$.

In general, connectedness type properties of a TVG may differ very much from those of a TVs. Let it suffice to mention that a 'genuine' TVG $E$ may have a lot of discrete vector subspaces and that the spaces $v(E) \subset c(E) \subset E$ may be drastically different. Thus, e.g., if $N$ is the Nevanlinna class over the unit disc in C, then $v(N)$ is the Smirnov class, and $v(N) \neq c(N) \neq N$ (see [8]).

In this paper, our main object of interest are the metrizable TVLG's $\omega(\eta)=$ $\left(\omega, \tau_{\eta}\right)$, where $\omega$ is the vector lattice of all real sequences, $\eta$ is a strictly positive submeasure on (all subsets of) $\mathrm{N}$, and $\tau_{\eta}$ is the topology of convergence in submeasure $\eta$. The spaces $\omega(\eta)$ have recently been introduced in [4], but most of the attention was paid to their largest topological vector subspaces, $\lambda_{0}(\eta)=v(\omega(\eta))$.

For a general metrizable TVLG $E$ that is nestedly complete (see Section 2), we give in Theorem 2.2 a description of $c(E)$ in terms of $\varepsilon$-chainability, and prove that $c(E)$ is a closed ideal in $E$. Next, in Theorem 2.3, we show that various types of connectedness for $E$ are mutually equivalent; this is very close to a result of H. Weber [10, Th. 5.10]. For the special case of $E=\omega(\eta)$, we give in Theorem 3.3 a description of $c(\omega(\eta))$, and in Theorem 3.4 we prove that $\omega(\eta)$ is (pathwise) connected iff $\eta$ is core-nonatomic (see Section 3). Furthermore, in Theorem 3.5 we characterize those submeasures $\eta$ for which $\lambda_{0}(\eta)=c(\omega(\eta))$. In Section 4, we give some relevant examples. Finally, in Theorem 5.1, we give an extension of Theorem 3.4 to the TVG's $\omega(X, \eta)$ of vector-valued sequences.

We owe our original inspiration to the paper of J. W. Roberts [8], but the only trace of it that remained can now be seen in Remark 2.4. The final shape of our results has been influenced very much by the work of H. Weber [10], where connectedness type properties were studied for general uniform lattices. In particular, it prompted us to state some of our original results for $\omega(\eta)$ as results concerning general metrizable TVLG's in Section 2.

We thank Professor Hans Weber for calling our attention to his paper, and for a very nice (and simple) argument showing that the core-nonatomicity of $\eta$ is necessary for $\omega(\eta)$ to be connected. We used his idea, in somewhat modified forms, in the proofs of Theorems 3.3, 3.4, and 5.1.

\section{General results}

We recall that, given $\varepsilon>0$, a subset $A$ of a metric space $(M, \rho)$ is called $\varepsilon$-chainable if for every pair of points $x, y \in A$ there exists an $\varepsilon$-chain in $A$ from $x$ to $y$, i.e., a finite sequence $v_{0}, \ldots, v_{k}$ in $A$ such that $x=v_{0}, y=v_{k}$, and $\rho\left(v_{i}, v_{i+1}\right)<\varepsilon$ for $0 \leqslant i<k$. If $A$ is connected, then it is $\varepsilon$-chainable for 
every $\varepsilon>0$; cf. [10, Lemma 5.6]. (Given $x \in A$, the set of those $y \in A$ that are $\varepsilon$-chainable with $x$ is closed and open.) The converse is easily seen to hold when $A$ is compact. For basic facts about connected spaces and sets, we refer to [6]. In particular, let us note that a Hausdorff space is pathwise connected iff it is arcwise connected [6, 6.3.12].

In all of this section, $E$ is a metrizable TVLG with the topology defined by a monotone $F G$-norm $\|\cdot\|$ (actually, $E$ could be any metrizable topological lattice group). We shall say that $E$ is nestedly complete if, whenever $\left(\left[x_{n}, y_{n}\right]\right)_{n \in \mathrm{N}}$ is a decreasing sequence of order intervals in $E$ with $y_{n}-x_{n} \rightarrow 0$, there is a (unique) point $z$ such that $z \in\left[x_{n}, y_{n}\right]$ for all $n$. Evidently, $z$ is then the limit of either of the sequences $\left(x_{n}\right)$ and $\left(y_{n}\right)$, as well as the sup of the $x_{n}$ 's, and the inf of the $y_{n}$ 's.

Clearly, if $E$ is $\sigma$-Dedekind complete or (topologically) intervally norm complete, then it is nestedly complete. It is important to note that the nested completeness is strictly weaker than what is known in the literature as the $(\sigma$-)interpolation property (see $[11$, Ch. $20, \S 146])$. E.g., $C[0,1]$ is nestedly complete but does not have the interpolation property. We refer the reader to [3] for more information on the nested completeness.

A crucial result for what follows is Proposition 2.1 below or, more specifically, the straightforward construction presented in its proof. It is used in a more or less direct way to derive all the other results of the paper. In particular, it is a basic ingredient of the proof of Theorem 2.3 which is a somewhat refined version of a result due to $H$. Weber [10, Th. 5.10] (although we chose not to state it in the full generality of that paper). Formally, the refinement is minor, being just a replacement of the assumption of the interpolation property of $E$ by its nested completeness. However, it makes our Theorem 2.3 applicable, e.g., to all intervally complete TVLG's $E$ which was not the case for the original result.

Proposition 2.1. Let $w, x \in E, w<x$, be such that for any $\varepsilon>0$ there is an $\varepsilon$-chain in $E$ from $w$ to $x$. Then for any pair of points $y, z$ with $w \leqslant y<z \leqslant x$ and any $\varepsilon>0$ there is a strictly increasing $\varepsilon$-chain from $y$ to $z$.

Moreover, if $E$ is nestedly complete, then for any pair $y, z$ as above there exists a strictly increasing continuous function $\varphi:[0,1] \rightarrow E$ with $\varphi(0)=y$ and $\varphi(1)=z$.

Proof. Fix $\varepsilon>0$. We first show, following [10, Lemma 5.7], that there is an increasing $\varepsilon$-chain $v_{0}, \ldots, v_{k}$ from $w$ to $x$. By assumption, there is an $\varepsilon$-chain $w_{0}, \ldots, w_{k}$ from $w$ to $x$. Let $v_{i}^{\prime}=\left(w_{i} \vee w\right) \wedge x$ and $v_{i}=\sup _{0 \leqslant j \leqslant i} v_{j}^{\prime}$ $(0 \leqslant i \leqslant k)$. Then, by Birkhoff's inequalities (see [1]), $\left|v_{i+1}-v_{i}\right| \leqslant \mid v_{i+1}^{\prime}-$ $v_{i}^{\prime}|\leqslant| w_{i+1}-w_{i} \mid$, and the $v_{i}$ 's are as required. Let now $w \leqslant y<z \leqslant x$ and 
$u_{i}=\left(v_{i}+y-w\right) \wedge z$. Then $u_{0}, \ldots, u_{k}$ is an increasing $\varepsilon$-chain from $y$ to $z$, and we can make it strictly increasing by deleting every term that is equal to an earlier term.

We now prove the second assertion. Fix a sequence $\left(\varepsilon_{n}\right)$ of positive numbers with $\varepsilon_{n} \rightarrow 0$. Applying inductively the first part, we find for each $n \in \mathrm{N}$ a strictly increasing $\varepsilon_{n}$-chain $C_{n}=\left\{v_{n, 0}, v_{n, 1}, \ldots, v_{n, k_{n}}\right\}$ from $y$ to $z$ so that $C_{n} \subset C_{n+1}$. The latter inclusion means that for each $0 \leqslant i<k_{n}$ there is a segment $v_{n+1, p}, v_{n+1, p+1}, \ldots, v_{n+1, q}(p<q)$ of the $\varepsilon_{n+1}$-chain $C_{n+1}$ that joins $v_{n, i}$ and $v_{n, i+1}$. Next, for each $n$ choose a sequence $T_{n}=\left\{0=t_{n, 0}<t_{n, 1}<\right.$ $\left.\cdots<t_{n, k_{n}}=1\right\}$ in $[0,1]$ so that for $i$ and $p<q$ as above, $t_{n, i}=t_{n+1, p}<$ $\cdots<t_{n+1, q}=t_{n, i+1}$. Clearly, this can be done so that the union $T$ of all the $T_{n}$ 's is dense in [0,1]. For each $n$ denote $\delta_{n}:=\min \left\{t_{n, i+1}-t_{n, i}: 0 \leqslant i<k_{n}\right\}$.

Define a function $\psi: T \rightarrow E$ by $\psi\left(t_{n, i}\right)=v_{n, i}$ for $n \in \mathrm{N}$ and $0 \leqslant i \leqslant k_{n}$. Clearly, $\psi$ is strictly increasing. Moreover, if $t, t^{\prime} \in T$ and $\left|t-t^{\prime}\right|<\delta_{n}$, then $\left\|\psi(t)-\psi\left(t^{\prime}\right)\right\|<2 \varepsilon_{n}$. (Assume that $t<t^{\prime}$ and observe that there is $i$ such that either $t_{n, i} \leqslant t<t^{\prime} \leqslant t_{n, i+1}$ or $t_{n, i-1} \leqslant t<t_{n, i}<t^{\prime} \leqslant t_{n, i+1}$.)

Finally, extend $\psi$ to a function $\varphi:[0,1] \rightarrow E$ as follows: Let $s \in[0,1]$. For every $n$, let $t_{n}$ be the largest point in $T_{n}$ that is $\leqslant s$, and $t_{n}^{\prime}$ be the smallest point in $T_{n}$ that is $\geqslant s$. Then the sequences $\left(t_{n}\right)$ and $\left(t_{n}^{\prime}\right)$ in $T$ are, respectively, increasing and decreasing, and $t_{n}^{\prime}-t_{n} \rightarrow 0$. Hence also $\left\|\psi\left(t_{n}^{\prime}\right)-\psi\left(t_{n}\right)\right\| \rightarrow 0$. Making use of the nested completeness, we now let $\varphi(s)=\sup _{n} \psi\left(t_{n}\right)=$ $\lim _{n} \psi\left(t_{n}\right)$. Then $\varphi$ is as required.

THEOREM 2.2. $c(E)$ is a closed vector sublattice of E. Moreover, if $E$ is nestedly complete, then $c(E)$ consists of all elements $x \in E$ such that for each $\varepsilon>0$ there is an increasing $\varepsilon$-chain in $E$ from 0 to $|x|$, and $c(E)$ is an ideal in $E$.

In consequence, if $E$ is nestedly complete, so is $c(E)$.

Proof. Since the closure of a connected set is again connected, $c(E)$ is closed. The rest of the first assertion follows from the continuity of the maps $(x, y) \rightarrow x+y, x \rightarrow t x(t \in \mathrm{R})$ and $x \rightarrow|x|$.

If $x \in c(E)$, then also $|x| \in c(E)$. Since $c(E)$ is connected, for each $\varepsilon>0$ there is an $\varepsilon$-chain from 0 to $|x|$ which, by Proposition 2.1, can be chosen to be increasing.

Now, assume that $E$ is nestedly complete. If $x$ satisfies the required condition, $y \in E$ and $|y| \leqslant|x|$ then, by Proposition 2.1 again, there are continuous curves joining 0 with $y^{+}$, and 0 with $y^{-}$. It follows that $y \in c(E)$, in particular, $x \in c(E)$. 
THEOREM 2.3. If $E$ is nestedly complete, then the following are equivalent.

(a) Whenever $w, x \in E$ and $w<x$, then for each $\varepsilon>0$ there is a strictly increasing $\varepsilon$-chain $v_{0}, v_{1}, \ldots, v_{k}$ from $w$ to $x$.

(b) Whenever $y, z \in E$ and $y<z$, then there exists a strictly increasing and continuous function $\varphi:[0,1] \rightarrow E$ with $\varphi(0)=y$ and $\varphi(1)=z$.

(c) Every order interval in $E$ is pathwise connected.

(d) Every solid subset of $E$ is pathwise connected.

(e) E is connected.

In consequence, if $E$ is connected, then all the balls in $E$ are pathwise connected.

Proof. By Proposition 2.1, (a) implies (b), and (e) implies (a). The implications (b) implies (c), (c) implies (d), and (d) implies (e) are easy (or trivial).

Remark 2.4. For the case where $E$ is norm complete, it is worth pointing out an alternative and somewhat simpler argument (inspired by [8]) proving that if (a) holds, then every order interval in $E$ is connected. It is enough to show that for any $z \in[w, x] \subset E$ there is a continuum $K$ such that $w, z \in K \subset[w, x]$. Assume, as we may, that $w<z$, and let the sequence $\left(\varepsilon_{n}\right)$ and the $\varepsilon_{n}$-chains $C_{n}$ be as in the proof of Proposition 2.1 for $y=w$. Let

$$
K=\overline{\bigcup_{n} C_{n}} \quad \text { and } \quad D_{n}=\bigcup_{i=0}^{k_{n}-1}\left[v_{n, i}, v_{n, i+1}\right] \quad(n \in \mathrm{N}) .
$$

Note that every order interval $\left[v_{n, i}, v_{n, i+1}\right]$ is of diameter $<\varepsilon_{n}$. Moreover, $C_{n} \subset D_{n}$ and $D_{n+1} \subset D_{n}$ for every $n$. It follows that $K \subset \bigcap_{n} D_{n}$, whence $K$ is totally bounded. Since $K$ is also closed, it is compact, and it is easily seen to be $\varepsilon$-chainable for each $\varepsilon>0$. Hence $K$ is a continuum, and $w, z \in K \subset$ $[w, z] \subset[w, x]$.

\section{The special case of $\omega(\eta)$}

Let $\mathscr{P}(\mathrm{N})$ denote the family of all subsets of $\mathrm{N}$. A function $\eta: \mathscr{P}(\mathrm{N}) \rightarrow[0, \infty]$ is called a submeasure on $\mathrm{N}$ if $\eta(\emptyset)=0, \eta(A) \leqslant \eta(B)$ whenever $A \subset B$, and $\eta(A \cup B) \leqslant \eta(A)+\eta(B)(A, B \in \mathscr{P}(\mathrm{N}))$. It is said to be (strongly) nonatomic if for every $\varepsilon>0$ there is a finite cover (or partition) $A_{1}, \ldots, A_{k}$ of $\mathrm{N}$ such that $\eta\left(A_{i}\right) \leqslant \varepsilon$ for each $i$. Note that then $\eta(A)=0$ for all finite sets $A \subset \mathrm{N}$.

A submeasure $\eta$ on $\mathrm{N}$ is said to be core-nonatomic if for every $\varepsilon>0$ there is a finite cover (or partition) $A_{0}, A_{1}, \ldots, A_{k}$ of $\mathrm{N}$ such that $A_{0}$ is a finite set and 
$\eta\left(A_{i}\right) \leqslant \varepsilon$ for $i=1, \ldots, k$. (See [4] for more details; also see Example 4.1 below.)

In all of this section, $\eta$ is a strictly positive submeasure on $\mathrm{N}$ (that is, one with $\eta(A)>0$ whenever $A \neq \emptyset)$, and $\omega(\eta)=\left(\omega, \tau_{\eta}\right)$ is the TVLG associated to $\eta$ that was already defined in the Introduction. A convenient monotone $F G$ norm defining the topology $\tau_{\eta}$ is given by

$$
\|x\|_{\eta}:=\inf \{\varepsilon>0: \eta(s(x, \varepsilon)) \leqslant \varepsilon\} \quad \text { for } \quad x=(x(j)) \in \omega,
$$

where

$$
s(x, \varepsilon)=\{j:|x(j)|>\varepsilon\} \quad(\varepsilon \geqslant 0) ;
$$

$s(x)=s(x, 0)$ is the support of $x$. Let $\tau_{p}$ denote the topology of coordinatewise convergence in $\omega$. Since $\left(\omega, \tau_{p}\right)$ is complete and $\tau_{\eta}$ is stronger than $\tau_{p}$, $\omega(\eta)$ is nestedly complete. Hence, in particular, all the results of the preceding section are applicable to $\omega(\eta)$.

We recall that if $\eta$ is lower semicontinuous, that is, $\eta\left(A_{n}\right) \uparrow \eta(A)$ whenever $A_{n} \uparrow A$, then $\omega(\eta)$ is complete (see [4, Fact 7.3]); in general, $\omega(\eta)$ need not even be intervally complete, see Example 4.3. Clearly,

$$
\lambda_{0}(\eta)=v(\omega(\eta))=\left\{x \in \omega: \lim _{t \rightarrow 0}\|t x\|_{\eta}=0\right\}
$$

is an ideal in $\omega$. For the following description of $\lambda_{0}(\eta)$, see [4, Fact 7.4].

Proposition 3.1. If $x \in \omega$, then $x \in \lambda_{0}(\eta)$ iff $\eta(s(x, r)) \rightarrow 0$ as $r \rightarrow \infty$.

It is also worth noting that $\omega(\eta)=\lambda_{0}(\eta)$ iff $\tau_{\eta}=\tau_{p}$ iff $\eta$ is order continuous, i.e., $\eta\left(A_{n}\right) \rightarrow 0$ whenever $A_{n} \downarrow \emptyset$ (cf. [4], Th. 7.16(a) and its proof).

In order to state our results in a concise form, we associate with $\eta$ another submeasure $\eta^{\circ}$ on $\mathrm{N}$ by defining, for every $A \subset \mathrm{N}, \eta^{\circ}(A)$ as the infimum of all those $\varepsilon>0$ for which there is a finite cover (or partition) $B_{1}, \ldots, B_{k}$ of $A$ such that $\eta\left(B_{i}\right) \leqslant \varepsilon$ for each $i$. Equivalently, $\eta^{\circ}(A)=\inf \max \left\{\eta\left(B_{1}\right), \ldots, \eta\left(B_{k}\right)\right\}$, where the infimum is taken over all finite covers (or partitions) of $A$. We collect some simple observations concerning $\eta^{\circ}$ in the proposition below, where we also use a strictly positive submeasure $\eta^{*}$ on $\mathrm{N}$ defined by $\eta^{*}(A)=$ $\sup _{n \in A} \eta(\{n\})$.

Proposition 3.2. The following hold.

(a) $\eta^{\circ} \leqslant \eta^{\circ} \leqslant \eta$ and $\eta^{\prime}(A)=\eta^{\circ}(A)$ for all finite sets $A \subset \mathrm{N}$.

(b) $\eta^{\circ}(A \cup B)=\max \left\{\eta^{\circ}(A), \eta^{\circ}(B)\right\}$ for all $A, B \subset \mathrm{N}$; likewise for $\eta$.

(c) $\left(\eta^{\circ}\right)^{\circ}=\eta^{\circ}$.

(d) If $\eta$ is order continuous, then $\eta^{\circ}=\eta$.

(e) $\eta$ is core-nonatomic iff $\eta^{\circ}$ is order continuous, and in that case $\eta^{\circ}=\eta$. 
For a set $A \subset \mathrm{N}$, we let $e_{A}$ denote the characteristic function of $A$, viewed as a sequence of zeros and ones. The main result of this section is the following characterization of $c(\omega(\eta))$, very much like that of $\lambda_{0}(\eta)$ given in Proposition 3.1.

THEOREM 3.3. The connected component $c(\omega(\eta))$ of the origin in the TVLG $\omega(\eta)$ consists of all $x \in \omega$ such that $\eta^{\circ}(s(x, r)) \rightarrow 0$ as $r \rightarrow \infty$; that is, $c(\omega(\eta))=\lambda_{0}\left(\eta^{\circ}\right)$.

Proof. In view of Theorem 2.2, $c(\omega(\eta))$ is a closed ideal in $\omega(\eta)$.

Let $x \in c(\omega(\eta))$. Then also $|x| \in c(\omega(\eta))$, and we may assume that $x \geqslant 0$. Fix any $\varepsilon>0$. By Theorem 2.2, there is an increasing $\varepsilon$-chain $v_{0}, v_{1}, \ldots, v_{k}$ from 0 to $x$. Let $u_{i}=v_{i}-v_{i-1}$ for $i=1, \ldots, k$. Then, for each $i, u_{i} \geqslant 0$ and $\left\|u_{i}\right\|_{\eta}<\varepsilon$ so that writing $B_{i}=s\left(u_{i}, \varepsilon\right)$ one also has $\eta\left(B_{i}\right)<\varepsilon$; moreover, $x=u_{1}+\cdots+u_{k}$. If $j \in \mathrm{N} \backslash\left(B_{1} \cup \cdots \cup B_{k}\right)$, then $0 \leqslant x(j)=u_{1}(j)+\cdots+$ $u_{k}(j) \leqslant k \varepsilon$. It follows that $\eta^{\circ}(s(x, k \varepsilon))<\varepsilon$.

To prove the converse direction, we may again assume that $x>0$. Fix any $\varepsilon>0$, and let $r>0$ be such that $\eta^{\circ}(s(x, r))<\varepsilon$. Denote $B_{0}=\mathrm{N} \backslash s(x, r)$ and choose a partition $B_{1}, \ldots, B_{k}$ of $s(x, r)$ so that $\eta\left(B_{i}\right)<\varepsilon$ for each $1 \leqslant i \leqslant k$. Denote $z_{i}=x e_{B_{i}}$ for $0 \leqslant i \leqslant k$. Then $\left\|z_{i}\right\|_{\eta} \leqslant \eta\left(s\left(z_{i}\right)\right)<\varepsilon$ for $1 \leqslant i \leqslant k$, while $z_{0} \in l_{\infty} \subset \lambda_{0}(\eta)$. Hence, choosing $m \in \mathrm{N}$ large enough one has $\left\|m^{-1} z_{0}\right\|_{\eta}<\varepsilon$. Set $z_{i}=m^{-1} z_{0}$ for $i=k+1, \ldots, k+m=n$. Then the elements $v_{0}=0, v_{i}=z_{1}+\cdots+z_{i}$ for $i=1, \ldots, n$, form an increasing $\varepsilon$-chain from 0 to $x$. By Theorem 2.2, $x \in c(\omega(\eta))$.

Our next two results are easy consequences of the theorem above.

TheOREM 3.4. The TVLG $\omega(\eta)$ is connected (and hence has all the other properties listed in Theorem 2.3) if and only if $\eta$ is core-nonatomic.

Proof. If $\eta$ is core-nonatomic, then $\eta^{\circ}$ is order continuous so that the condition in Theorem 3.3 is satisfied for every $x \in \omega$. Conversely, if $c(\omega(\eta))=\omega$ then, in particular, $x=(j)_{j \in \mathrm{N}} \in c(\omega(\eta))$. Hence, by Theorem 3.3, $\eta^{\circ}(\{n, n+$ $1, \ldots\}) \rightarrow 0$ as $n \rightarrow \infty$. Thus $\eta^{\circ}$ is order continuous or, equivalently, $\eta$ is core-nonatomic.

THEOREM 3.5. $\lambda_{0}(\eta)=c(\omega(\eta))$ iff $\eta$ satisfies the following condition.

(*) Whenever $\left(A_{n}\right)$ is a sequence of subsets of $\mathrm{N}$ with $A_{n} \downarrow \emptyset$ and $\eta^{\circ}\left(A_{n}\right) \rightarrow$ 0 , then also $\eta\left(A_{n}\right) \rightarrow 0$.

Proof. Assume that $\lambda_{0}(\eta)=c(\omega(\eta))$, and let a sequence $\left(A_{n}\right)$ be as specified above. Define $x=(x(j)) \in \omega$ by $x(j)=n$ for $j \in A_{n} \backslash A_{n+1}(n \in \mathrm{N})$. Then, by Theorem 3.3, $x \in c(\omega(\eta))$. Hence, by the assumption, $x \in \lambda_{0}(\eta)$ and, by Proposition 3.1, $\eta\left(A_{n}\right)=\eta(s(x, n-1)) \rightarrow 0$. 
Conversely, let $\eta$ satisfy condition (*). If $x \in c(\omega(\eta))$ and $A_{n}=s(x, n)$ for each $n \in \mathrm{N}$ then, by Theorem 3.3, the sequence $\left(A_{n}\right)$ is as required in $(*)$, hence $\eta\left(A_{n}\right) \rightarrow 0$. It follows that $x \in \lambda_{0}(\eta)$.

REMARKs 3.6. (a) It is fairly obvious that a submeasure $\eta$ is core-nonatomic and satisfies condition $(*)$ iff it is order continuous iff $\lambda_{0}(\eta)=c(\omega(\eta))=\omega$.

(b) For a core-nonatomic submeasure $\eta$, it is easy to see that solid sets are pathwise connected also in the space $\omega(\eta)$ of complex sequences.

(c) It is worth pointing out that for no strictly positive submeasure $\eta$ on $\mathrm{N}$ is the associated Fréchet-Nikodym metric space $(\mathscr{P}(\mathrm{N}), \eta)$ connected. (Simply note that the family of sets containing 1 is closed-open.)

\section{Examples}

ExAmPLe 4.1. A very large class of core-nonatomic strictly positive submeasures on $\mathrm{N}$ can be obtained as follows: Take any sequence $\mathbf{F}=\left(F_{n}\right)$ of finite nonempty sets with union $\mathrm{N}$ such that $\left|\left\{n:\left|F_{n}\right|=k\right\}\right| \leqslant 2^{\lambda k}$ for some $\lambda \geqslant 0$ and all $k \in \mathrm{N}$, and put

$$
\bar{d}_{\mathbf{F}}(A)=\sup _{n} \frac{\left|A \cap F_{n}\right|}{\left|F_{n}\right|}, \quad d_{\mathbf{F}}(A)=\limsup _{n \rightarrow \infty} \frac{\left|A \cap F_{n}\right|}{\left|F_{n}\right|} \quad \text { for } A \subset \mathrm{N} .
$$

Then $d_{\mathbf{F}}$ is a nonatomic submeasure (see [5, Th. 2.1]), and $\bar{d}_{\mathbf{F}}$ is a corenonatomic submeasure (cf. [4, Fact 4.3])). Moreover, $\bar{d}_{\mathbf{F}}$ is strictly positive and lower semicontinuous, but it is not order continuous. In consequence, the TVLG $\omega\left(\bar{d}_{\mathbf{F}}\right)$ is complete and $\lambda_{0}\left(\bar{d}_{\mathbf{F}}\right) \neq c\left(\omega\left(\bar{d}_{\mathbf{F}}\right)\right)=\omega$ (by Theorem 3.4). In the 'classical' case, i.e., when $F_{n}=\{1, \ldots, n\}$, we denote the corresponding submeasures simply by $\bar{d}$ and $d$.

Examples 4.2. (a) Let a function $f: \mathrm{N} \rightarrow(0, \infty)$ be such that $\lim _{\sup } f(n)$ $>0$. Then the submeasure $\sigma_{f}$ on $\mathrm{N}$ defined by $\sigma_{f}(A)=\sup f(A)$ satisfies (*) (in fact, $\left(\sigma_{f}\right)^{\circ}=\sigma_{f}$ ) but is not core-nonatomic. Therefore, $\lambda_{0}\left(\sigma_{f}\right)=$ $c\left(\omega\left(\sigma_{f}\right)\right) \neq \omega$.

(b) Let $\eta_{0}(A)=1$ when $A \neq \emptyset, \eta_{0}(\emptyset)=0$. Also, let $N_{0}$ denote the set of even integers in $\mathrm{N}$, and $N_{1}=\mathrm{N} \backslash N_{0}$. Then the submeasure $\eta$ given by $\eta(A)=\eta_{0}\left(A \cap N_{0}\right)+\bar{d}\left(A \cap N_{1}\right)$ is not core-nonatomic and does not satisfy $(*)$. Therefore, $\lambda_{0}(\eta) \neq c(\omega(\eta)) \neq \omega$.

Note that both $\sigma_{f}$ and $\eta$ are lower semicontinuous.

EXAmple 4.3. Here we give a class of core-nonatomic submeasures $\eta$ for which the space $\lambda_{0}(\eta)$ (and, a fortiori, $\omega(\eta)$ ) is not even intervally complete.

Let $\mu$ be any nonatomic submeasure on $\mathrm{N}$ with $\mu(\mathrm{N})=1$, and let $v$ be a submeasure on $\mathrm{N}$ defined by $v(A)=\sup _{n \in A} a_{n}$, where $a_{n}>0$ and $\sum_{n} a_{n}<$ 
$\infty$. Then $\eta=\max \{\mu, \nu\}$ is a core-nonatomic strictly positive submeasure on $\mathrm{N}$ and, obviously, it is not order continuous. Therefore, $\lambda_{0}(\eta) \neq c(\omega(\eta))=\omega$. Now, consider the sequence $\left(e_{n}\right)$ of standard unit vectors in $\omega$. Since $\left\|e_{n}\right\|_{\eta} \leqslant$ $\eta\left(s\left(e_{n}\right)\right)=\eta(\{n\})=a_{n}$, the series $\sum_{n} e_{n}$ is Cauchy in $\omega(\eta)=\left(\omega,\|\cdot\|_{\eta}\right)$. Suppose it converges in $\omega(\eta)$. Then it also converges coordinate-wise so that its sum has to be $e_{\mathrm{N}}$ (the constant 1 sequence). Therefore, letting $z_{n}=e_{\mathrm{N}}-$ $\sum_{i \leqslant n} e_{i}$, we have $\left\|z_{n}\right\|_{\eta} \rightarrow 0$. However, for any $0<\varepsilon<1, \eta\left(s\left(z_{n}, \varepsilon\right)\right) \geqslant$ $\mu\left(s\left(z_{n}, \varepsilon\right)\right)=1$ so that $\left\|z_{n}\right\|_{\eta}=1$ for every $n$; a contradiction. We have thus shown that the order interval $\left[0, e_{\mathrm{N}}\right] \subset \lambda_{0}(\eta)$ is not complete.

Let us additionally observe that if $\mu=d_{\mathbf{F}}$, where $\mathbf{F}=\left(F_{n}\right)$ is as in Example 4.1, and the $a_{n}$ 's above are chosen so that $a_{n} \leqslant \bar{d}_{\mathbf{F}}(\{n\})$ for each $n$, then $\eta \leqslant \bar{d}_{\mathbf{F}}$. Moreover, if $\left(A_{n}\right)$ is any sequence in $\mathscr{P}(\mathrm{N})$ with $A_{n} \downarrow \emptyset$, then $\eta\left(A_{n}\right) \rightarrow 0$ iff $\bar{d}_{\mathbf{F}}\left(A_{n}\right) \rightarrow 0$ (cf. [4, Fact 3.5]). Therefore, $\lambda_{0}(\eta)=\lambda_{0}\left(\bar{d}_{\mathbf{F}}\right) \neq$ $c(\omega(\eta))=c\left(\omega\left(\bar{d}_{\mathbf{F}}\right)\right)=\omega$.

\section{An extension to vector sequences}

Let $X=(X,\|\cdot\|)$ be a nonzero Banach space, and let $\omega(X, \eta)$ denote the TVG $\omega(X)$ of all $X$-valued sequences with the topology $\tau_{\eta}$ of convergence in submeasure $\eta$. The same formula as in the scalar case (with an obvious modification) gives an $F G$-norm $\|\cdot\|_{\eta}$ defining $\tau_{\eta}$. Clearly, if $x=\left(x_{j}\right) \in \omega(X)$, then $\left\|\left(x_{j}\right)\right\|_{\eta}=\left\|\left(\left\|x_{j}\right\|\right)\right\|_{\eta}$.

THEOREM 5.1. Let $\eta$ be a lower semicontinuous strictly positive submeasure on $\mathrm{N}$. Then the following are equivalent.

(a) $\eta$ is core-nonatomic.

(b) Every open ball in $\omega(X, \eta)$ is pathwise connected.

(c) $\omega(X, \eta)$ is connected.

Proof. Since $\eta$ is lower semicontinuous, the closed balls $B(0, r)(r>0)$ in $\omega(X, \eta)$ are easily seen to be closed in the topology $\tau_{p}$ of coordinate-wise convergence. Moreover, $\left(\omega(X), \tau_{p}\right)$ is complete and $\tau_{p} \leqslant \tau_{\eta}$. Hence $\omega(\eta)$ is complete (cf. [4, Fact 7.3]).

(a) implies (b). We start with an observation. Consider an open ball $K(y, r)$ in $\omega(X, \eta)$. Take any $z \in K(y, r)$ and fix any $\varepsilon>0$. By (a), there is a partition $A_{0}, A_{1}, \ldots, A_{k}$ of $\mathrm{N}$ with $A_{0}$ finite and $\eta\left(A_{i}\right)<\varepsilon$ for $1 \leqslant i \leqslant k$. Then the $\varepsilon$-chain $v_{0}, v_{1}, \ldots, v_{k}$ from $y$ to $z$, constructed as in the proof of Theorem 3.3, is such that $\left\|y-v_{i}\right\|_{\eta} \leqslant\|y-z\|_{\eta}$ for each $i$. (In the construction, one may assume $y=0$.)

Now, let $K(w, r)$ be an open ball in $\omega(X, \eta)$. Fix any $x \in K(w, r)$ and a sequence $\left(\varepsilon_{n}\right)_{n \geqslant 0}$ of positive numbers such that $\|w-x\|_{\eta}+\sum_{n} \varepsilon_{n}=$ : $r^{\prime}<r$. Using the observation made above and proceeding as in the proof of 
Proposition 2.1, one constructs $\varepsilon_{n}$-chains $C_{n}=\left\{v_{n, 0}, v_{n, 1}, \ldots, v_{n, k_{n}}\right\}$ from $w$ to $x$ so that $C_{n} \subset C_{n+1}$ and, additionally, $\|w-v\|_{\eta} \leqslant\|w-x\|_{\eta}+\left(\varepsilon_{0}+\cdots+\varepsilon_{n-1}\right)$ for each $v \in C_{n}$.

Next, define the sets $T_{n}$ and $T$, the constants $\delta_{n}$, and the function $\psi$ as in the proof just mentioned. Then, as easily seen, $\left\|\psi(t)-\psi\left(t^{\prime}\right)\right\|_{\eta}<2\left(\varepsilon_{n+1}+\varepsilon_{n+2}+\right.$ $\cdots)$ whenever $t, t^{\prime} \in T$ and $\left|t-t^{\prime}\right|<\delta_{n}$. Thus $\psi$ is uniformly continuous on $T$, and its range is contained in the closed ball $B\left(w, r^{\prime}\right)$. To finish, extend $\psi$ by continuity to all of $[0,1]$. In consequence, $K(w, r)$ is pathwise connected.

That (b) implies (c) is obvious. To verify that (c) implies (a), take any sequence $x=(x(j))$ with $\|x(j)\| \rightarrow \infty$. By (c), given $\varepsilon>0$, there is an $\varepsilon$-chain $v_{0}, \ldots, v_{k}$ from 0 to $x$. Define $u_{i}, B_{i}$ and $A_{0}$ as in the proof of Theorem 3.3. Then $x=u_{1}+\cdots+u_{k}, \eta\left(B_{i}\right)<\varepsilon$, and $\|x(j)\| \leqslant\left\|u_{1}(j)\right\|+$ $\cdots+\left\|u_{k}(j)\right\| \leqslant k \varepsilon$ for each $j \in A_{0}$. It follows that $A_{0}$ is finite, and by disjointizing the $B_{i}$ 's we conclude that (a) is satisfied.

Remark 5.2. The implication (a) implies (b) remains valid when $X=$ $(X,\|\cdot\|)$ is an $F$-space (see [7]), and so does (c) implies (a) provided that $X$ admits an equivalent $F$-norm, say $\|\cdot\|^{\prime}$, which is unbounded, i.e., $\sup _{x \in X}\|x\|^{\prime}=$ $\infty$. We do not know however if (and how) one could prove that (c) implies (a) without that assumption (which is the case, e.g., for the $F$-space $L_{0}$ of all measurable functions on $[0,1]$, with the topology of convergence in Lebesgue measure, see $[9,9.2 .2])$.

\section{REFERENCES}

1. Aliprantis, C., and Burkinshaw, O., Locally Solid Riesz, Spaces with Applications to Economics, 2nd ed., Math. Surveys and Monographs 105, Amer. Math. Soc., Providence, RI 2003.

2. Drewnowski, L., Topological vector groups and the Nevanlinna class, Funct. Approx. Comment. Math. 22 (1994), 25-39.

3. Drewnowski, L., On nestedly complete topological vector lattices, in preparation.

4. Drewnowski, L., and Labuda, I., Solid sequence F-spaces of $L_{0}$-type over submeasures on N, Illinois J. Math. 53 (2009), 623-678.

5. Drewnowski, L., and Łuczak, T., On nonatomic submeasures on N, Arch. Math. (Basel) 91 (2008), 76-85.

6. Engelking, R., General Topology, Monogr. Mat. 60, PWN - Polish Sci. Publ., Warszawa 1977.

7. Kalton, N. J., Peck, N. T., and Roberts, J. W., An F-space sampler, London Math. Soc. Lecture Note 89, Cambridge Univ. Press, Cambridge 1984.

8. Roberts, J. W., The component of the origin in the Nevanlinna class, Illinois J. Math. 19 (1975), 553-559.

9. Rolewicz, S., Metric Linear Spaces, PWN - Polish Scientific Publishers \& D. Reidel Publishing Co., Warszawa \& Dordrecht 1984.

10. Weber, H., On modular functions, Funct. Approx. Comment. Math. 24 (1996), 35-52. 
11. Zaanen, A. C., Riesz spaces II, North-Holland Math. Library 30, North-Holland, Amsterdam 1983.

FACULTY OF MATHEMATICS AND COMPUTER SCIENCE

A. MICKIEWICZ UNIVERSITY

UMULTOWSKA 87

61-614 POZNAŃ

POLAND

E-mail: drewlech@amu.edu.pl

E-mail: nawrocki@amu.edu.pl 